\title{
A proactive collaborative workshop approach to supporting student preparation for graduate numerical reasoning tests
}

Eleanor Lingham, Maths Learning Centre, De Montfort University, Leicester, UK. Email: elingham@dmu.ac.uk

Ann Baughan, Careers and Employability, De Montfort University, Leicester, UK. Email: abaughan@dmu.ac.uk

\begin{abstract}
Numerical competency and reasoning skills are of high importance and high concern to graduate recruiters. The use of numerical reasoning tests in graduate recruitment is increasing. Many students are unaware of the prevalence of these tests, and the need for refreshment and practice of numerical skills. We describe a stand-alone workshop that is jointly run by the Maths Learning Centre, and the Careers and Employability Service at De Montfort University. This workshop helps students to proactively prepare for these tests by providing test information, preparation tips and signposting to further maths and career support. The workshop's main feature is a testing activity that is run individually and for small groups. Findings suggest that these workshops have been effective and are popular with students.
\end{abstract}

Keywords: employability, graduate, recruitment, numerical, psychometric.

\section{Introduction}

This paper describes an innovative approach to supporting students in graduate recruitment numerical test preparation, based on:

- Close collaboration and team workshop design and presentation, by the Maths Learning Centre (MLC) and Careers and Employability (C\&E) at De Montfort University (DMU);

- Development of a bespoke workshop, 50\% mathematics, 50\% test preparation tips and skills;

- Using individual and team testing to assess and develop successful numerical techniques;

- Signposting to further mathematics and careers support;

- Follow-up access to an online graduate recruitment test programme that measures progress and suggests areas for development.

\section{Background}

Hughes, Sheen and Birkin (2013, p.17) report that "Amongst the cross-cutting skills identified by employers as being in greatest demand, competency in mathematics frequently appears high up in lists of desirable characteristics of graduates." Another report finds that general numeracy is in short supply among graduates and postgraduates in many industries (Docherty and Fernandez, 2014, p.7). It is therefore unsurprising that the use of numerical reasoning tests has increased from $33 \%$ of graduate recruiters in 2004 (CFE Research, 2004) to at least 52\% of employers in 2014 (CFE Research, 2014). (The survey found that $67.4 \%$ of graduate recruiters use psychometric testing, and that $77.2 \%$ of these used numerical reasoning tests.) 
In 2012, the Maths Learning Centre observed an increased number of students who were coming for mathematics support for graduate recruitment tests. Often these students came looking for help at 'the eleventh hour' - that is, after they had been invited to take part in an online numerical reasoning test (often with a 48 hour deadline). Maths preparation and support at this late stage was inevitably of limited benefit to the student and, arguably, an inefficient and ineffective use of staff time within an open mathematics drop-in support session.

It was clear that a new approach was needed to support students in their numerical reasoning test preparation. Students needed to be informed about the prevalence of numerical reasoning tests, and the need to refresh their numerical reasoning skills as an integral part of their graduate recruitment preparation. The importance of extensive question practice needed to be emphasised, and a proactive approach to problem acquisition and solution needed to be encouraged. Accordingly, the Maths Learning Centre approached Careers and Employability to work together on an approach to address these issues.

\section{Overall design of workshop}

Although various solutions were possible, we needed an approach with minimal cost that would be relatively quick to implement, and which would reach as many students as possible. We also hypothesised that students attending open drop-ins were there because of wanting face-to-face support (otherwise they would have found online resources already). Although we invested in online resources for follow-on support, we considered that initial face-to-face contact was a key factor in motivating students to do more preparation.

\subsection{Aims}

There were two main aims in the creation of the numerical reasoning preparation workshops:

- Encourage a proactive approach among students, and early extensive revision of numerical reasoning skills;

- Provide signposting to the full range of mathematics and careers support that is available in the institution, and suggest ways to maximise effective and efficient access to it.

Achievement of these aims would benefit students in their search for graduate jobs and also staff within support services by stream-lining this particular aspect of work.

\subsection{Delivery}

To maximise the number of students who could access the numerical reasoning workshops, we decided to develop a 'standalone' format that would run monthly (more often in the autumn term) at a time outside of timetabled lectures for most students. Hence, it usually runs on Wednesday lunchtime and is 1.5 hours in duration. Places are open to all students and graduates and can be booked through the online student portal. We limit spaces to 40 per session, to promote smooth running of the testing activities.

The workshops have two presenters (one apiece from MLC and C\&E) so that we can each lead on our own speciality (although we are both able to present solo on occasion, as needs arise).

We begin with a description of graduate recruitment tests and an explanation for why companies use them, while emphasising their prevalence. We then run a testing activity, where participants have ten minutes to individually complete a seven question test. In the following ten minutes, they tackle the same test in teams of three or four. We then discuss the solutions in plenary, and 
provide hints and tips for successful test preparation. Further maths and careers support options are then highlighted. We also promote the verbal reasoning workshops run by colleagues. Directions are given to the online graduate recruitment test programme to which the institution has subscribed. The workshop concludes with a question and answer session, and feedback evaluation sheet.

\subsection{Testing activity}

When designing the workshop, we decided that including a short individual testing activity would give students a chance to self-assess their numerical reasoning skills in a supportive environment. In order to avoid deterring anxious students, we do not mention the individual testing activity in promotional literature, and when introducing the test, we take care to do so in a positive encouraging way.

To maximise the usefulness of the test, we designed seven multiple choice questions of industrylevel standard, that cover a broad range of numerical reasoning skills (percentages, ratios etc.) as well as a broad range of question contexts (engineering, business etc.). We chose a time limit of ten minutes to put students under some pressure, but not excessive pressure. We use an online countdown timer to encourage an exam-like atmosphere.

The individual test is followed by a ten minute team activity where students work on the same questions in groups of three or four. This is a chance for them to explain their work to others, or see how other people problem-solved. We assign the teams using a brief (random) maths activity, and encourage students to see the team 'test' as an opportunity for them to self-assess their use of graduate employability skills such as assertiveness, leadership, teamwork. The fact that they are working with peers that they have never met before is very useful here. At the end of the activity, teams submit a solution sheet so that we can assess their work and announce the winning team.

In the solutions section of the workshop, we focus on questions where the teams have struggled to get the right answer. As well as explaining the techniques, we emphasise faster ways of answering questions. Due to time constraints, and the range of maths skills among participants, we speak broadly about the questions and distribute full solutions at the end of the session.

\subsection{Evaluation}

We use paper evaluation forms to gather feedback on sessions. As part of this, we ask participants to self-assess their numerical reasoning confidence and knowledge on a scale of 1 (very low) to 10 (very high), at the start and end of the workshop. We also gather comments about the session and ask the student where they heard about the session. Forms are collected at the end 'in exchange' for a copy of the slides and solutions. Analysis of the participant population as a whole can now be undertaken annually using data obtained from the online booking system. We aim to gain further insight into the diversity of students accessing the workshops and their employment outcomes.

\section{Outcomes}

Participants in the last two years have reported increased knowledge and confidence for numerical reasoning as a result of attending the workshop. The increase reported by participants the previous year is shown in brackets. For 2014/15, the average increase in knowledge was 4.1 to 7.9 [ 4.4 to 7.3], and for confidence from 3.5 to 7.2 [3.8 to 6.6]. This data reflects virtually the entire participant population as our collection method ensures almost $100 \%$ completion of feedback forms. 
Attendance on these standalone workshops has been high (113 in 2013/14 and 96 in 2014/15). The latter figure excludes over 30 participants attending workshops that are now embedded into C\&E employability programmes.

In terms of future enhancements, due to student demand, and the timing of graduate recruitment, we plan to run a similar number of standalone sessions in 2015/16 but frontload more into the autumn term. Also, the online booking system has enabled us to look at diversity data for participants, and gives us the potential to tailor advertising to reach low participation groups such as white males $(66 \%$ of participants are female compared with a DMU profile of $57 \%)$. We are looking at the possibility of running specific workshops to target some of these under-represented groups (for example, technology students). One option would be to run an in-faculty workshop as part of one of the university's 'Enhancement Weeks'.

Finally, the MLC has seen fewer 'last minute' numerical reasoning test students. In terms of the overall aims of setting up the workshops, this suggests that students have become more aware of the prevalence of numerical reasoning testing and the need for extensive practice. It also suggests that signposting to the full range of maths support that is available is becoming more effective. C\&E are very satisfied with the partnership and the establishment of a programme to provide a high quality, proactive psychometric testing support service to students across the university.

\section{References}

Docherty, D. and Fernandez, R. (2014). Career Portfolios and the Labour Market for Graduates and Postgraduates in the UK. National Centre for Universities and Business.

Hughes, T., Sheen, J. and Birkin, G. (2013). Industry graduate skills needs. National Centre for Universities and Business.

CFE Research, (2004). The AGR Graduate Recruitment Survey. Summer Review.

CFE Research, (2014). The AGR Graduate Recruitment Survey. Summer Review. 